important roles in injury prevention, not only by treating injured patients, but also by addressing the community conditions that increase the risk for injury. In order to build injury prevention into the healthcare system, healthcare organisations can benefit from models that bridge community prevention and health service delivery.

Objective In this session, participants will learn about Prevention Institute's Community-Centred Health Home (CCHH) model to promote systems change and active involvement in community advocacy. The vision of a community-centred health home takes models such as community oriented primary care and the medical home a step further by encouraging health care institutions to take an active role in strengthening their surrounding community. Results When a Chinatown resident was struck and killed by a car while crossing the street in Oakland, California, Asian Health Services (AHS) staff became aware of pedestrian safety as a health issue in their community. AHS engaged in the CCHH steps of inquiry, analysis, and action and collaborated with several community groups, business owners, and city planning agencies to instal a "scramble" crosswalk to stop vehicle traffic and allow pedestrians to safely cross in all directions. Early data showed the "scramble" reduced car-pedestrian incidents by as much as fifty percent. A larger community transportation and planning project also emerged out of the coalition to further address environmental factors impacting pedestrian safety.

Conclusions The CCHH model is being piloted in several states in the United States. The model can be applied in other counties to advance the integration of injury prevention into the healthcare system.

\section{PROFILE OF SELF-INFLICTED HARM IN LOS ANGELES BY RACE/ETHNICITY, 2001-2010}

${ }^{1} \mathrm{~S}$ Bazargan-Hejazi, ${ }^{2} \mathrm{~A}$ Ahmadi, ${ }^{3}$ Elham Rahmani, ${ }^{1} \mathrm{M}$ Mojtahedzadeh, ${ }^{1} \mathrm{D}$ Pan. ${ }^{1}$ Charles $R$. Drew University; ${ }^{2}$ Kermanshah University of Medical Sciences and Karolinska Institute; ${ }^{3}$ Boston University

\subsection{6/injuryprev-2016-042156.450}

Background Self-Inflicted Harm is one of the major causes of morbidity and mortality. Previous studies have shown that there are different patterns of SIH among different racial groups. This study aims to describe SIH admission rates and patterns among different racial groups in Los Angeles and to evaluate whether admission rates have changed over a ten year period (20012010).

Methods Data for this retrospective study includes all cases of hospital admission in Los Angeles with SIH based on the ICD-9: E950-E958 for 2001-2010. ICD-9 codes for schizophrenia, episodic mood disorders, depressive, anxiety, personality adjustment, sexual and gender identity disorders, as well as alcoholism and substance abuses were used.

Results A total of 2459 patients with SIH were identified. African Americans(AA) had the highest ten year average of SIH rate $(11.6 / 100,000)$ followed by Caucasians(CC) $(8.44 / 100,000)$, Latinos(LT) (6.21/100,000) and Asians(AS) (1.64/100,000). The SIH admission rates remained steady throughout the 10 year study period $(\mathrm{P}$ value $=0.3064)$ and none of the racial groups showed a significant change $(\mathrm{P}$ value $\mathrm{CC}=0.2445, \mathrm{AA}=0.2120$, $\mathrm{LT}=0.6898, \mathrm{AS}=0.1237)$. Median age of SIH was significantly lower in Latinos $(\mathrm{LT}=28$ versus $\mathrm{CC}=43, \mathrm{AA}=39$ and $\mathrm{AS}=$ 40 ( $\mathrm{P}$ value $<0.001)$. Overall, episodic mood disorders where the most common psychiatric comorbidity among all racial groups. However, AAs had the lowest probability of episodic mood disorder comorbidity $(\mathrm{CC}=55.87 \%$, $\mathrm{AA}=39.52 \%$, $\mathrm{LT}=41.84 \%$, AS $=47.66 \%, \mathrm{P}$ value $<0.001)$ and the highest probability of schizophrenia comorbidity $(\mathrm{CC}=10.47 \%$, $\mathrm{AA}=20.84 \%, \mathrm{LT}=8.00 \%, \mathrm{AS}=10.49 \%$, P value $<0.001)$.

Conclusions SIH admission rates as well as the gap between different racial groups have remained steady during the study period which can raise concerns about the effectiveness of programs aimed at decreasing $\mathrm{SIH}$ admission rates and/or reducing interracial disparity.

\section{Posters Monday 19.9.2016 Child and Adolescent Safety}

\section{Post Mon 1.7}

\section{INJURY INCIDENCE OF CHILDREN BELOW 5 YEARS OF AGE, IN A COMMUNITY HEALTH DIVISION IN SRI LANKA}

${ }^{1}$ Indrakantha Welgama, ${ }^{2}$ Piyanga Chandradasa, ${ }^{3}$ Samath Dharmaratne. 'Office of the Regional Director of Health Services, Colombo, Sri Lanka; ${ }^{2}$ Office of the Medical Officer of Health, Homagama, Sri Lanka; ${ }^{3}$ Department of Community Medicine, Faculty of Medicine, University of Peradeniya, Sri Lanka

\subsection{6/injuryprev-2016-042156.451}

Background Main objective of this study was to find the incidence and characteristics of injuries within the past 1 year period, suffered by children under 5 years of age in this selected community health division in Sri Lanka.

Methods This was a community based descriptive, cross sectional study covering a randomly selected area of the Homagama Community Health division in Colombo District. The population was children under 5 years who were permanent residents of that area, and a single child under the age of 5 years was randomly selected from each eligible family, following community household visits. A structured, pre-tested, interviewer administered questionnaire with trained interviewers and pictorial support material, was used for data collection, over 3 months period in 2013.

Results Of 2136 respondents, 552 (25.84\%) had a history of injury during the past 1 year. Of the 1077 (50.42\%) males, $26.92 \%$ have had injuries, while $24.74 \%$ of females too had injuries. Children aged 3 to 4 years were mainly vulnerable for injuries, while $35.3 \%$ of children have reported only one significant (as perceived by parents/guardians) injury during the past year. Common injury types were abrasions (45.82\%), lacerations $(17.06 \%)$ and contusions (9.7\%). Of those reporting injuries, $4.35 \%$ had suffered fractures. Most of the causes for these injuries were falls $(75.5 \%)$, followed by falling objects, animal attacks and burns. $67.5 \%$ of injuries had occurred at home while $17.4 \%$ had occurred in the home garden.

Conclusions From the findings of this study, it could be concluded that the incidence of injuries amongst under 5 year old children in the community in this area was $25.84 \%$ and also that the incidence was similar between both sexes. The commonest injuries were abrasions and lacerations while falls, falling objects, animal attacks and burns were the main causes of these injuries. Home and home garden were the common environments in which these injuries had occurred. 